\title{
HEDGEHOG: Drone Perching on Tree Branches with High-Friction Origami Spines
}

\author{
Steffen Kirchgeorg, and Stefano Mintchev, Member, IEEE
}

\begin{abstract}
The collection of environmental and biodiversity data is essential to manage, preserve and restore forests, but this task remains challenging due to the inaccessibility of these ecosystems. Compared to human intervention, aerial robots can access tree canopies, but their limited flight time and noise continue to stall widespread application. To address this challenge, we present a perching mechanism which allows small drones to rest on overhanging branches and extend their mission while remaining silent. We developed an origami spine with two folding flaps containing a layer of high-friction material. When the spine engages with a branch, the flaps open and conform to irregular branch surfaces generating sufficient friction to support the weight of a drone. With HEDGEHOG, a drone integrating multiple spines on a protective cage, we demonstrated its application in a controlled indoor as well as in a forest environment. We modelled the perching strategy and measured the effects of materials and geometric parameters on the drone's perching performance. By leveraging interactions with nature, our drone can perch on tree branches with diameters up to $86 \mathrm{~mm}$ and inclined up to $\pm 15^{\circ}$ and potentially remain in the canopy for extended periods of time to acquire data or monitor returning wildlife.
\end{abstract}

Index Terms-Aerial systems, mechanism design, perching drone, origami technology.

\section{INTRODUCTION}

$\mathbf{T}$ HE preservation and restoration of forests has recently gained fast growing interest due to the variety of benefits, known as ecosystem services, they provide to billions of people on Earth [1,2]. While forest management needs to be addressed on a global scale, it is a local challenge requiring detailed social and ecological knowledge of the site [3]. However, the lack of large temporal and spatial data hinders a better scientific understanding of biodiversity, microclimatic variables and their correlation in forest ecosystems [4]. For a holistic approach to forest management, such understanding is indispensable.

Forests and high tree canopies remain hard and expensive to access for scientists as fixed towers need to be built or experienced climbers are required [4]. Drones on the other

Manuscript received: September 3, 2021; Accepted November 11, 2021

This paper was recommended for publication by Editor Pauline Pounds upon evaluation of the Associate Editor and Reviewers' comments. This work was supported by the Swiss National Science Foundation through the Eccellenza Grant PCEFP2_186865.

The authors are with the Environmental Robotics Laboratory, Dep. of Environmental Systems Science, ETH Zurich, 8092 Zurich, Switzerland and with the Swiss Federal Institute for Forest, Snow and Landscape Research (WSL), 8903 Birmensdorf, Switzerland (e-mail: skirchgeorg@ethz.ch, smintchev@ethz.ch).

Digital Object Identifier (DOI): 10.1109/LRA.2021.3130378

(C2021 IEEE. Personal use of this material is permitted.

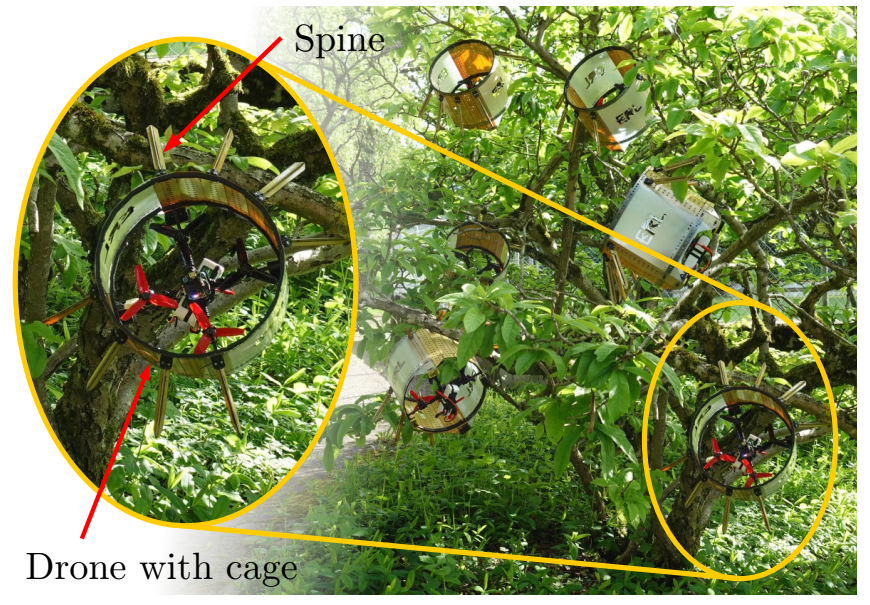

Fig. 1. HEDGEHOG perching on tree branches in the natural environment through high-friction origami spines.

hand can reach these high and remote areas rather easy, making them a perfect candidate for environmental monitoring. Yet, the limited flight time restricts the collection of environmental and biodiversity data over longer time periods. In addition, the sound of the unmanned aerical vehicle (UAV) can cause a vigilant or even fearful response for some wildlife, possibly scaring the animal away [5,6] and making their monitoring more difficult. By enabling UAVs to perch or rest - the ability of attaching to a surrounding structure and reduce power consumption - longer term environmental monitoring tasks could be achieved. The drone could silently monitor returning wildlife or carry out other data collection tasks while remaining within the canopy. After minutes or even hours, the drone could wake up and return to base or move on to a new monitoring site.

While small UAVs have already demonstrated flights within tree canopies [7-9], their small payloads demand for a perching mechanism and strategy that does not rely on heavy sensing and complex computation. Recent works focus mainly on perching on man-made structures rather than natural ones, that are intrinsically more complex, irregular and unpredictable. Several mechanisms for perching on vertical walls based on microspines [10-12], dry adhesive [13-15] or magnetic force [16] were developed. Some of the solutions can also be used to attach to horizontal structures, such as concrete ceilings $[17,18]$. With respect to irregular and round objects, cage grasp solutions have been developed. The grasping mechanism relies on either encircling most of the object $[19,20]$ or using an opposed grip technique [21]. For these solutions to be successful, a precise alignment of the grasping mechanism

This document is the accepted manuscript version of the following article: 
with the object is necessary, which can be challenging to achieve in a dense and cluttered environment or with limited computing power. With respect to natural structures, [22] achieved perching on leafs through electroadhesion. However, as the mechanism was implemented on an insect-size flying robot, the scalability as well as attachment to irregular surfaces through electroadhesion remain a challenge.

Directly addressing perching in trees, [21] developed a bistable gripper that can hold a load of up to $4.6 \mathrm{~N}$ but requires a small amount of energy to change states. [23] developed a passive microspine grapple that can engage with a branch and hold large weights. As it is connected to the UAV with a wire, the system requires open space on all sides of the branch to approach, engage and then rotate around the object to achieve a stable position below the grapple. The wire also increases the risk of unwanted entanglement with other surrounding structures during the perching process.

Tree branches and twigs are ideal overhanging structures that can be used for perching in the forest. To exploit these structures, we aim for a passive attachment mechanism that can be mounted on a small and lightweight aerial platform and does not require precise alignment with the perchable object. Therefore, we present HEDGEHOG, an aerial vehicle integrating High-friction origami spinEs for Drone perchinG on treE brancHes for envirOnmental monitorinG. The passive perching mechanism is based on an origami spine that, once in contact with the surface of a branch, creates sufficient friction to support the weight of the hanging platform, as shown in Fig. 1. After successful perching, the drone can take off as the spine releases from the object once the load is removed.

In the following sections, we will first describe and model the perching strategy employed by HEDGEHOG, followed by the design of the drone and the origami spine. We will then analyse how the friction coefficient and the main platform design parameters affect the drone's perching before optimising the opening force of the origami mechanism. Finally, we demonstrate successful perching of HEDGEHOG on inclined circular objects indoors as well as on tree branches in unstructured natural environments.

\section{WORKING PRINCIPLE}

Passive perching within tree canopies is posing several challenges and requirements. The perching device shall engage with and disengage from the environment without active manipulation, additional sensing or control algorithms as these are difficult to achieve in obstructed and moving environments [24]. In these scenarios, passive mechanisms may be beneficial and more robust than active strategies [23]. To access tree canopies, the mechanism needs to furthermore be compact, lightweight and the drone shielded to avoid damage during interactions with the surrounding environment.

In this section, we first present our perching strategy to address the mentioned challenges. Then, we explain the design of HEDGEHOG and the origami spine before describing the manufacturing of the origami mechanism.

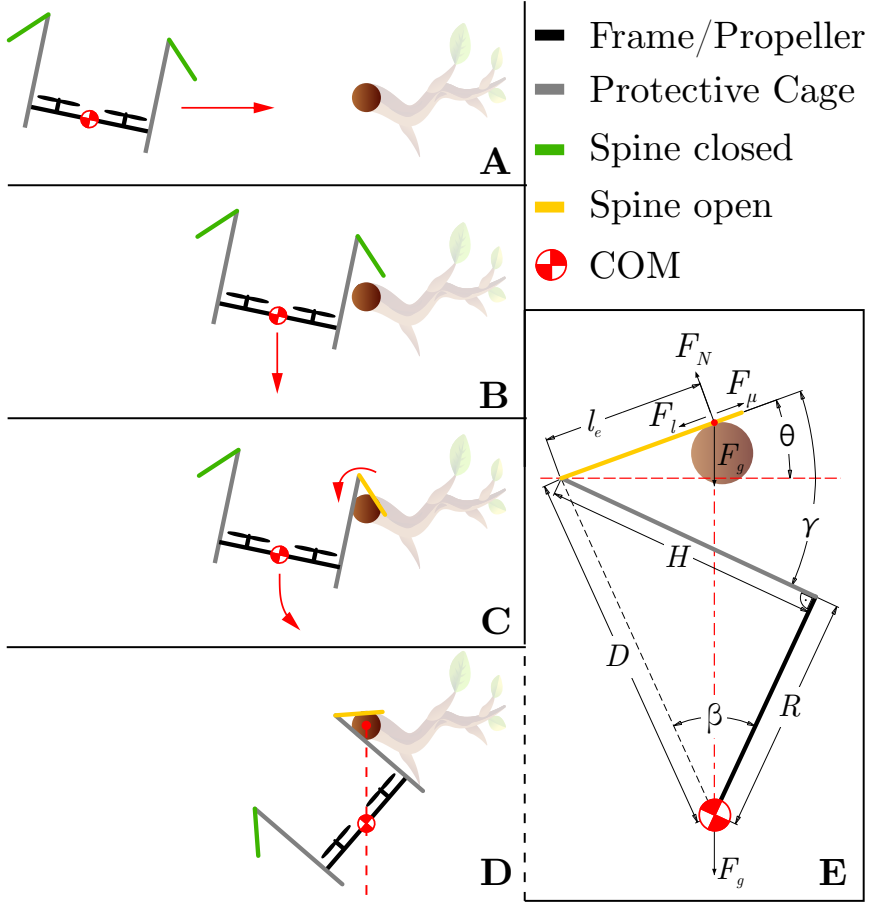

Fig. 2. Side section view of the perching sequence: (A) the drone approaches the object, (B) lowers itself onto the object until, (C) the spine engages and pivots around the point of engagement to reach (D) a stable perching position by aligning the COM below the point of rotation. (E) Free body diagram with forces acting on the drone and perching object.

\section{A. Perching strategy}

The proposed solution allows perching to a variety of overhanging structures by approaching from the top, side or partially from below the object. When approaching from the side, as depicted in Fig. 2, HEDGEHOG reaches the perching object laterally and can utilize its protective cage to touch the object for additional support prior to perching. The platform is then lowered onto the obstacle (Fig. 2B). Once the origami spine has made contact with the obstacle, the spine opens and a high-friction material is exposed (Fig. 2C). The spine starts to support HEDGEHOG and by reducing thrust, the platform transfers weight onto the spine, increasing friction with the obstacle. As the motor thrust is reduced, HEDGEHOG continues to pivot and align its center of mass (COM) below the obstacle (Fig. 2D). Once this is achieved, a stable perching position has been reached. No thrust is needed and no moment is induced as the COM is aligned vertically with the point of rotation.

The free-body diagram in Fig. 2E illustrates the forces during perching when a single spine is engaged (the model is extended for the engagement of two spines in B). The friction force $F_{\mu}$ counteracts the load $F_{l}$, which is a fraction of the weight of the system $F_{g}$ acting along the spine. Geometrical and equilibrium considerations show that the friction coefficient has to be greater than the tangent of the tilt angle,

$$
\mu>\tan (\theta),
$$

for the spine not to slip from the object (see Appendix A). Indeed, this behavior can be compared to a skier facing down a slope. On a gentle slope, the skier is not moving as the 
coefficient of friction between ski and snow is high enough to prevent sliding. However, at a certain slope angle the skier will start sliding. The angle at which sliding occurs can be calculated through eq. (1) with the friction coefficient between ski and snow. Compared to the skier, we want the spine not to slide, which means that the friction coefficient should be maximized and the "slope angle", in our case the tilt angle $\theta$ of the drone, minimized.

The first condition is achieved by adding high-friction material to the spines and designing them with compliant flaps that conform and maximize adhesion to surfaces even if they are uneven (see section II-B). Minimization of the tilt angle $\theta$ depends on the attachment point $l_{e}$ and the design parameters of HEDGEHOG (see Appendix A):

$$
\theta=\gamma-\beta+\arctan \left(l_{e} / D\right) .
$$

$\beta$ describes the mounting position of the spine given by height $H$ and radius $R$ from the COM, while $\gamma$ is the orientation of the spine with respect to the protective cage. The minimization of $\theta$ can be achieved by maximizing $\beta$ and minimizing $\gamma$. Former is limited by a minimum width of the platform for large enough propellers to create sufficient lift, and a maximum height to still remain compact for flying in dense environments. Reducing $\gamma$ directly limits the range of perchable branch sizes and should be avoided. Finally, the distance $l_{e}$ between the spine attachment on the cage and the branch contact increases the tilt angle and should be reduced whenever possible.

An extended the model for a simplified case of two symmetrically engaged spines is shown in Appendix B.

\section{B. Design of the platform and origami spine}

HEDGEHOG has a cylindrical protective cage to which we attach multiple origami spines that serve as the perching mechanism (Fig. 3A). A single spine is an origami mechanism consisting of two movable flaps attached to a center piece with flexural joints (Fig. 3B). These joints allow for independent rotation of each flap. The bottom surfaces of each flap are equipped with high-friction material. The flaps are normally in a closed state with a flap-to-flap opening angle $\kappa$ of $60^{\circ}$. The interference of the layers of high-friction material limits this angle. The flaps can open with center-to-flap opening angles $\alpha$ in the range $30^{\circ}<\alpha_{l, r}<180^{\circ}$. This combined with the compliant nature of the origami composite allows the flaps to adjust to irregular and inclined structures as shown in Fig. 3C. In the open state, the high-friction material is exposed and in contact with the environment, enabling the spine to exert a large friction force. Opening of the flaps is achieved by applying a load to the spine which presses the flaps against a surface. In our case, the load is the weight of HEDGEHOG, which also determines the holding force that the mechanism needs to sustain (Fig. 2E).

Multiple origami spines are integrated into a protective cage on HEDGEHOG to create a robust and redundant perching mechanism as shown in Fig. 3A. Besides the two states of the spine, each appendage can also pitch downward due to an additional flexible joint between the center piece and the attachment to the protective cage, illustrated in Fig. 3D. The benefit of this additional degree of freedom is illustrated in Fig. 3E. During perching, the spines adjacent to the contact spine should bend to avoid interference with the branch. This is especially important when perching on large diameter

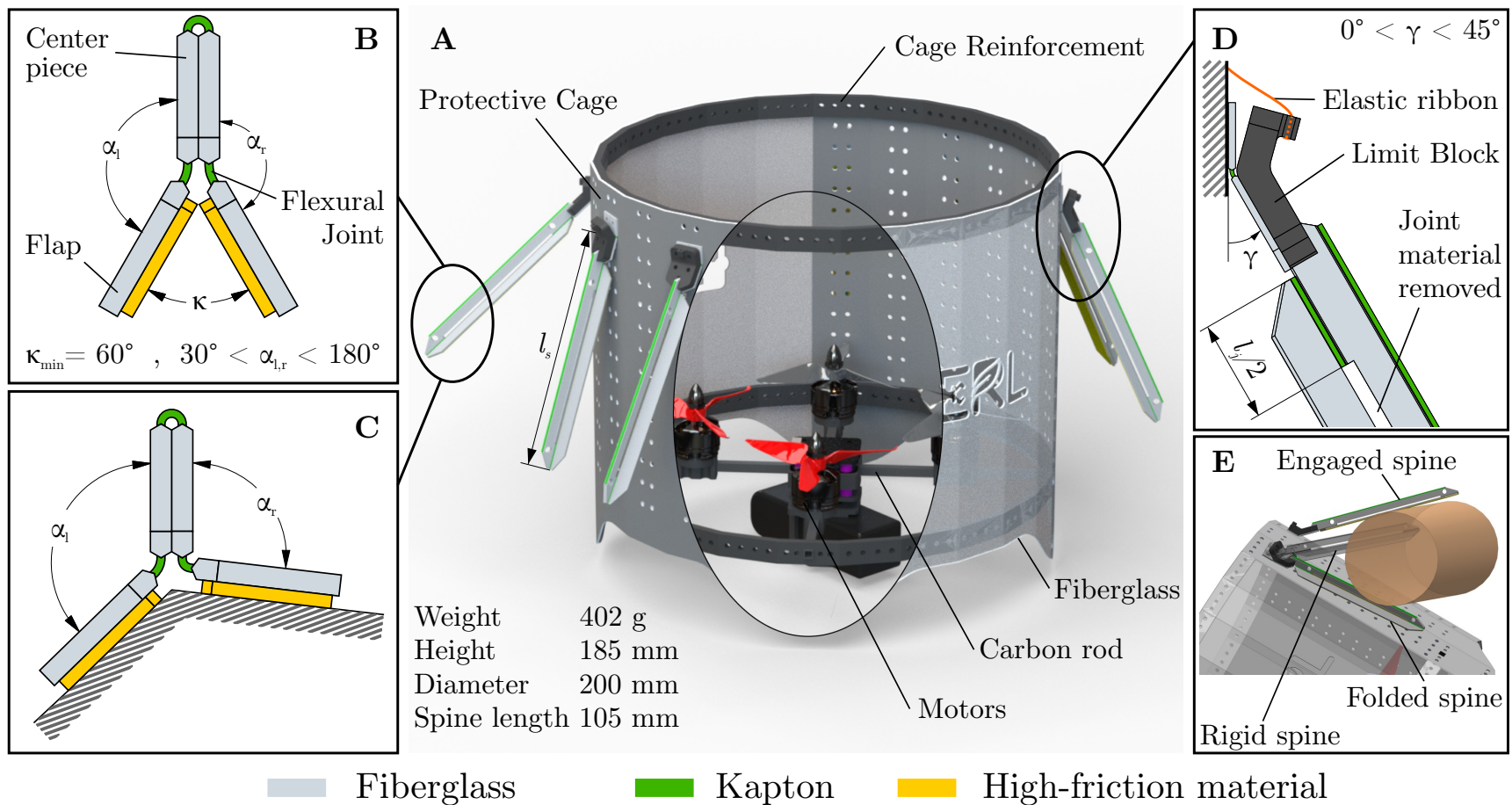

Fig. 3. Drawings and schematics of HEDGEHOG and the perching spines. (A) 3D model of the drone. Schematics of the origami spine: (B) closed with $\kappa_{\min }=60^{\circ}$, (C) engaged with origami flaps adjusting to the substrate and (D) the pitching mechanism that allows downward rotation in the range of $0^{\circ} \geq \gamma \geq 45^{\circ}$ with a default angle $\gamma=45^{\circ}$ achieved through the use of an elastic ribbon attached to the cage. (E) Illustration of the interference of branches with the spines if they can't fold under the branch. 


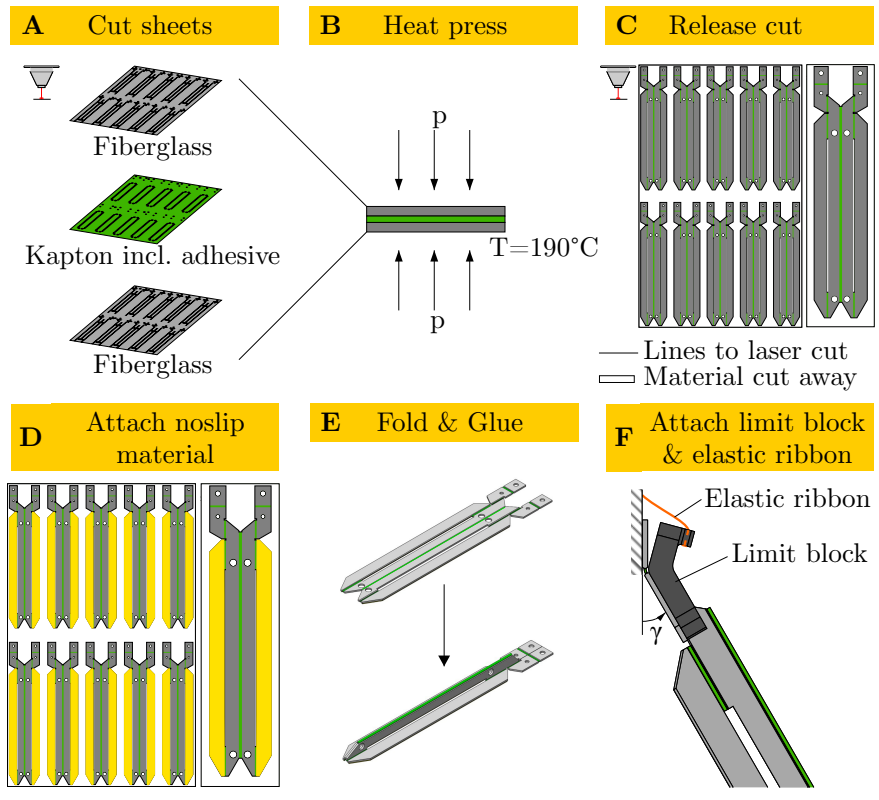

Fig. 4. Schematic of the manufacturing process of the origami spine and integration into the protective cage. (A) Cut fiberglass and Kapton (incl acrylic adhesive) sheets. (B) Heat press and bond the layers under pressure $p$ and $T=190^{\circ} \mathrm{C}$ for $t=1 \mathrm{~h}$ to a single component. (C) Release cut the component to bring forth the spine geometries. (D) Attach high-friction material to the flat sheet. (E) Fold the spines and bond together adjacent fiberglass layers with glue. (F) Attach limit blocks and elastic ribbons to achieve default position of $\gamma=45^{\circ}$ and enable downward rotation of the spine.

branches. The default position of the spine is set by using a limit block together with an elastic ribbon. The spines and elastic ribbon are attached with screws to a cylindrical protective cage made with fiberglass. The cage is reinforced with 3D-printed supports and mounted to the four carbon rods that hold the motors.

\section{Manufacturing}

The spine is based on origami technology and is made of two layers of fiberglass (FR-4-HF, $0.35 \mathrm{~mm}$ ) on the outside and a flexible layer of adhesive Kapton (DuPont Pyralux LF0111, $0.05 \mathrm{~mm}$ ) on the inside. The fiberglass thickness defines the structural strength as well as the compliance of the mechanism, while Kapton serves as a flexible joint material. In a first step (4A), the 2D design for all layers was cut from large sheets with a CO2 laser (Trotec Speedy 360). For the fiberglass layers, material was removed in the joint areas where, after bonding of all layers, adhesive Kapton serves as a joint. Only alignment holes had to be cut in the adhesive Kapton layer for the assembly. With these alignment holes and matching pins, all three layers were aligned and stacked on top of each other. Subsequently, the layers were bonded to a single composite in a heat press (Fontijne Presses LabManual 300), as shown in Fig. 4B. The composite thickness amounted to $0.85 \mathrm{~mm}$, making it stiff enough to withstand impacts while maintaining a compliant behaviour. This helps the flaps conform to surfaces to maximize surface contact.

To finalize the $2 \mathrm{D}$ origami structure, a laser cut was made to remove material from the composite to release and allow
TABLE I

FRICTION COEFFICIENT FOR MATERIAL COMBINATIONS OF FIBERGLASS OR DYCEM NON-SLIP WITH SMOOTH MDF AND PMMA.

\begin{tabular}{lcc} 
& Fiberglass & Dycem Non-slip \\
& $\mu_{f}$ & $\mu_{n}$ \\
\hline Smooth MDF & 0.21 & 0.96 \\
Poly(methyl methacrylate) (PMMA) & 0.44 & 1.75 \\
\hline
\end{tabular}

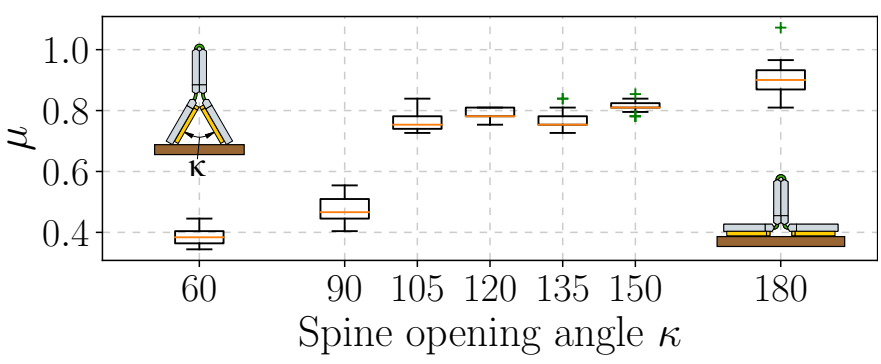

Fig. 5. Friction coefficient $\mu$ for different spine opening angles $\kappa$ on a flat piece of smooth MDF. During the test, the angle of the spine $\kappa$ is held in a fixed position with 3D printed parts not shown in the figure.

the movement of the joints (Fig. 4C). At this stage, the highfriction material was glued (Ethyl-2-cyanoacrylate) to the 2D geometry. The flat origami mechanism could now be folded into the 3D shape of the previously presented origami spine. Adjacent fiberglass layers were fixed together for the final shape with glue. The 3D printed limit block that prevents upward rotation beyond $\gamma=45^{\circ}$ was added with screws to the spine. The total weight of a single spine amounted to less than $5 \mathrm{~g}$. The two protective cage elements (Fig. 3A) for attaching the spines were also made as origami structures following the steps A to C in Fig. 4.

\section{RESUlts}

The chosen perching strategy is relying on friction and we therefore first characterize and maximize the friction coefficient of the spine. The geometric parameters of HEDGEHOG influence the second important parameter, the tilt angle during perching, which we assess thereafter. As successful perching requires the opening of the spine, we determine and optimize the opening force. Finally, we demonstrate perching of HEDGEHOG on inclined circular objects in a controlled indoor environment and on branches outdoor.

\section{A. Friction coefficient}

As summarized in eq. (1), successful perching requires to maximize the friction coefficient between the spine and the branch. For this reason, we added a layer of high-friction material (Dycem Non-slip) to the fiberglass surface of the spine flaps contacting the branch. Tested on smooth MediumDensity Fibreboard (MDF) and PMMA, the Dycem Nonslip material increased the coefficient of friction achievable using fiberglass alone by 4.5 and 3.9 times, respectively (see Table I). To maximize the friction coefficient, it is also important that the flaps open and conform to the perching surface. To test this hypothesis we characterized the friction coefficient on a flat surface of smooth MDF as a function 


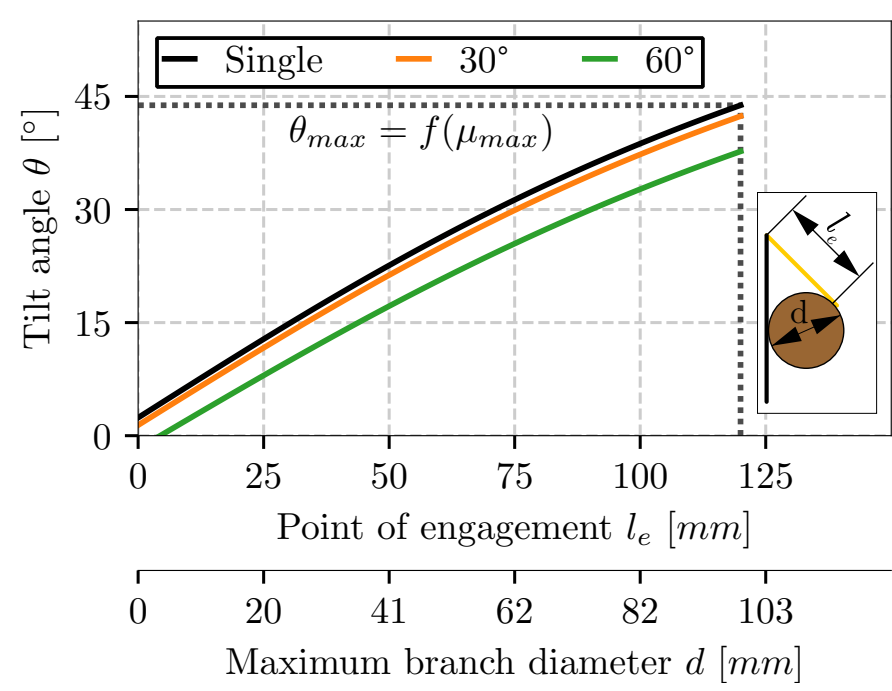

Fig. 6. Tilt angle of HEDGEHOG depending on the point of engagement $l_{e}$ of a single or two symmetrically engaged spines spaced $30^{\circ}$ and $60^{\circ}$ apart. Assuming a circular branch as an obstacle, distance $l_{e}$ can be converted to a branch diameter $d$.

of spine opening angle $\kappa$ in Fig. 5. The opening angle of the spine was fixed and the friction coefficient determined through inclined plane measurements. The friction coefficient increases by more than twofold from closed to open state. This is due to the contact material changing from mainly fiberglass at $60^{\circ}$ to the high-friction material at $180^{\circ}$ opening. If the spine can adjust its flaps to the substrate as shown in Fig. 3C, the friction coefficient and thus perching capabilities can be maximized.

\section{B. Tilt angle}

The other parameter that influences the perching success is the tilt angle $\theta$ (eq. (1)), that needs to be minimized. Theta is a function of the design parameters of the drone $(\gamma, \beta$ and $D)$, and the distance $l_{e}$ between the spine attachment on the cage and the branch contact (see eq. (2)).

The design of the drone is a trade-off between functional needs and the quest to minimize $\theta$. Aiming for a maximum platform weight of 500 grams, we used three inch propellers with T-Motor F1507 3800kV brushless DC motors to provide sufficient lift. This results in a radius $R=10 \mathrm{~cm}$ for HEDGEHOG. As a compromise between total platform size including the spines, reachable distance of the spine, perchable object diameter as well as the requirement to keep the angle $\gamma$ small, we set the spine length $l_{s}$ to $105 \mathrm{~mm}$ with $\gamma=45^{\circ}$. Together with a platform height of $185 \mathrm{~mm}$, we could mount the spine at $H=92 \mathrm{~mm}$ above the COM, yielding $\beta=42.6^{\circ}$. Based on eq. (2), Fig. 6 shows the tilt angle depending on the chosen design parameters and the point of engagement $l_{e}$ for a single engaged spine or two symmetrically engaged spines spaced apart by $30^{\circ}$ or $60^{\circ}$ (see appendix B). According to eq. (1), the maximum friction coefficient sets an upper boundary to the tilt angle. Considering a friction coefficient of 0.96 , the maximum tilt angle is $43.8^{\circ}$. This sets a theoretical limit to $l_{e}$ of $120 \mathrm{~mm}$, and therefore to the maximum branch size that can be perched on (see box in Fig. 6). With our spine length of $l_{s}=105 \mathrm{~mm}$, we do not reach the upper limit and the
A
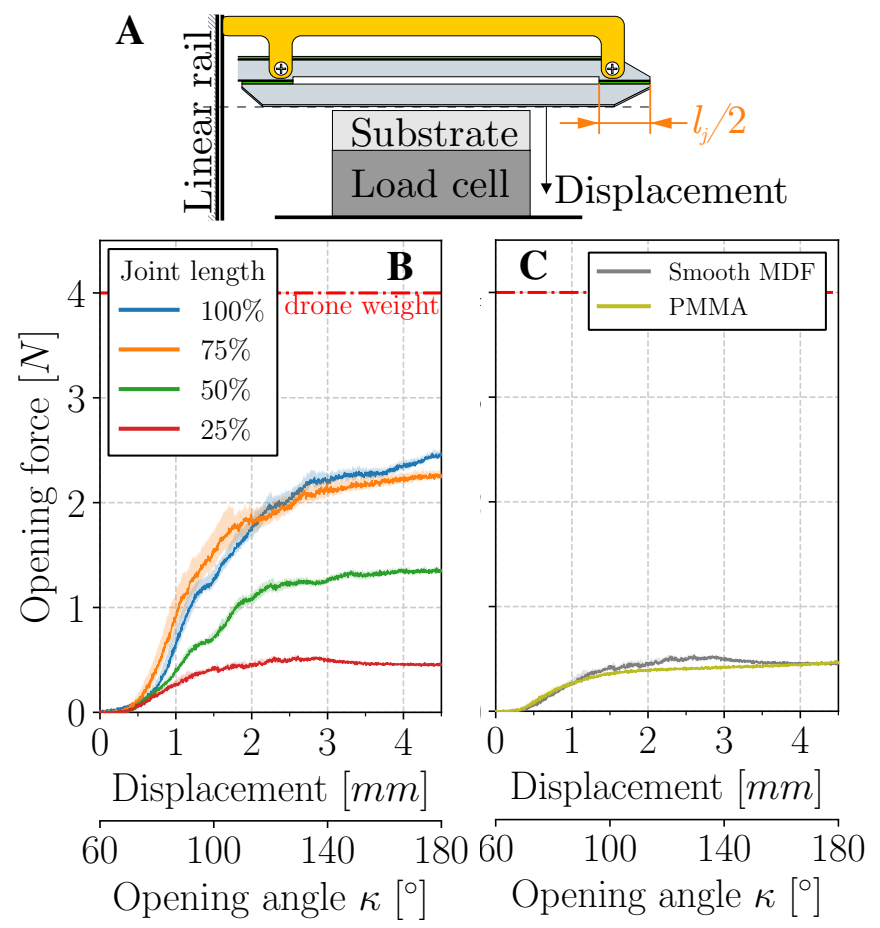

Fig. 7. Measurement of the opening force of the origami mechanism as a function of displacement, which is corresponding to the spine opening angle $\kappa$. (A) Experimental setup using a load cell. (B) Measurements against smooth $\operatorname{MDF}(\mu=0.96$ ) for different joint lengths. $100 \%$ joint length is equal to an absolute joint length of $l_{j}=72.5 \mathrm{~mm}$, (C) Measurements with two different materials: smooth MDF $\left(\mu_{n}=0.96\right)$ and PMMA $\left(\mu_{n}=1.75\right)$ with a fixed joint length of $25 \%\left(l_{j}=18.1 \mathrm{~mm}\right)$. Solid lines highlight the median values, while shaded areas show the $25^{\text {th }}$ and $75^{\text {th }}$ percentile.

maximum perchable diameter is $86 \mathrm{~mm}$. The engagement of two spines can result in a more stable perch as a reduction in tilt angle can be observed in Fig. 6.

\section{Opening Force Characterization}

Full opening of the origami spine mechanism is essential to achieve contact between the high-friction material and the environment and consequently support the weight of HEDGEHOG. Therefore we characterized the force required to achieve a $180^{\circ}$ opening angle $\kappa$ of the spine flaps (see Fig. 3B). First, we minimize the opening force as it will facilitate engagement. Second, we investigate how material roughness influences the opening of the spine.

The measurements of the opening force were obtained through a vertical displacement of the origami spine onto a substrate (see Fig. 7A). The displacement was achieved with a linear rail and the opening force acquired by a load cell on which the substrate was placed. For all measurement runs, ten cycles were recorded with the first being neglected as the origami joints underwent noticeable plastic deformation. As the spine opens under the weight of the platform, the opening force of the origami mechanism should be much lower than HEDGEHOG's weight. A small enough opening force can also allow two spines to engage at the same time. Different approaches can be taken to reduce the force necessary to deflect the joints of the flaps: (1) reduce the thickness of the joint material or (2) reduce the joint length. We decided to 

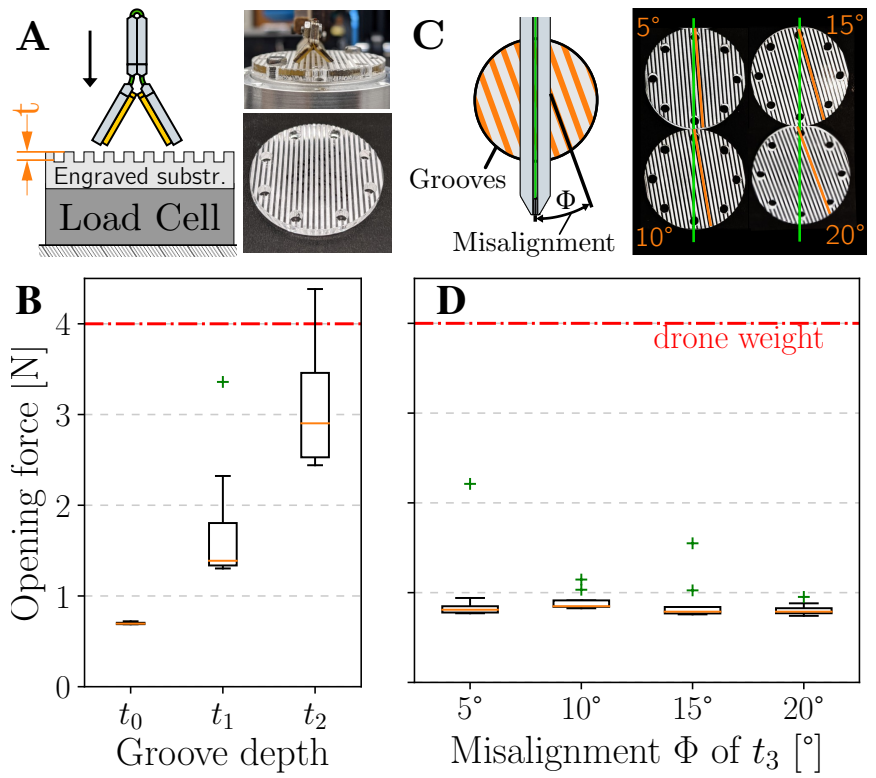

Fig. 8. Effect of corrugated surfaces on the opening force of the origami joint. (A) Experimental setup of spine being displaced onto the substrate mounted on the load cell and pictures of an engraved substrate. (B) Maximum opening force of the spine on a flat PMMA substrate $t_{0}=0 \mathrm{~mm}$ and two engraved PMMA substrates with groove depths of $t_{1}=0.08 \mathrm{~mm}$ and $t_{2}=0.14 \mathrm{~mm}$ that are aligned parallel with the spine. (C) Top view of the engraved substrate with angular misalignment $\Phi$ to the spine and pictures of engraved substrate with $\Phi=5^{\circ}-20^{\circ}$ misalignment. (D) Maximum opening force of the spine on an engraved PMMA substrate with groove depth $t_{3}=0.30 \mathrm{~mm}$ misaligned to the spine by the indicated angle.

reduce the joint length as we could use a single thickness Kapton material $(25 \mu \mathrm{m})$ and partially remove material from the center of the joint to achieve different joint lengths $l_{j}$ (see Fig. 3D). Fig. 7B shows median opening force versus displacement for different joint lengths. The measurements show a nearly proportional correlation between opening force and joint length. Starting from a base joint length of $l_{j}=72.5 \mathrm{~mm}$, indicated by $100 \%$, the force can be reduced to nearly onefifth with a joint length of $25 \%$. With a force $F_{\text {open }} \approx 0.5 \mathrm{~N}$, two spines can easily engage and open when considering HEDGEHOG's final weight of $4 \mathrm{~N}$. For subsequent tests and the final design, we therefore chose to move forward with the spine with $25 \%$ joint length $\left(l_{j}=18.1 \mathrm{~mm}\right)$.

Figure 7C shows little difference in opening force between smooth MDF and PMMA. The opening force is mainly used to bend and therefore store mechanical energy in the joints with negligible contribution from the friction force during opening. As tree branches usually exhibit non-smooth and even corrugated surfaces, we wanted to ensure that the spines are capable to open nonetheless. Therefore, we simulated non-smooth, corrugated surfaces through directional surface roughness by modifying three flat PMMA plates by engraving grooves of $0.75 \mathrm{~mm}$ width spaced the same amount apart. By varying the power of our laser, we achieved three depths besides the smooth sample (denoted with $t_{0}$ ): $t_{1}=0.08 \mathrm{~mm}$, $t_{2}=0.14 \mathrm{~mm}$ and $t_{3}=0.30 \mathrm{~mm}$ (see Fig. $8 \mathrm{~A}$ ). The probes were then mounted on the load cell and aligned so that the engraved pattern aligned parallel to the origami spine, as visible in Fig. 8A. The origami spine was pressed onto the
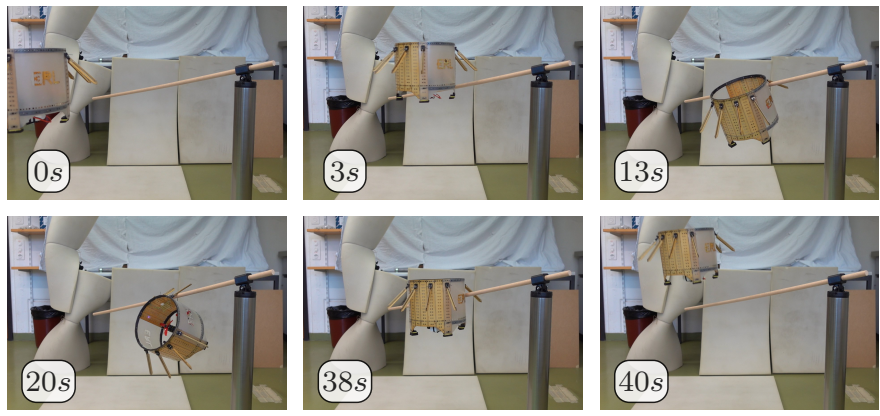

Fig. 9. Perching sequence on an inclined circular rod with a diameter of $15 \mathrm{~mm}$. (Top to bottom, left to right) HEDGEHOG is approaching from the side, positioning so that spines are slightly above the perching object, establishing a contact, reaching a stable perching position below the object, increasing thrust to disengage the spines and flying away.

substrates and the maximum amount of force required for opening of the mechanism was recorded. Fig. 8B indicates the statistical evaluation of the maximum opening force for nine measurement cycles per substrate.

A small corrugation (Fig. 8B, $t_{1}$ ) causes a strong increase in required opening force and for the medium groove depth (Fig. $\left.8 \mathrm{~B}, t_{2}\right)$ some measurements exceeded the platform weight, which means that the flaps would not open properly when mounted on the drone. For the roughest sample $\left(t_{3}\right)$, the outer fiberglass layer completely interlocked with the grooves on the corrugated surface and did not open even under high forces of up to $30 \mathrm{~N}$. However, with the same substrate being misaligned with respect to the spine (see Fig. 8C) by even small angles of $5^{\circ}$, the median opening force reduces and is only slightly higher than for the flat PMMA $\left(t_{0}\right)$, as evident from Fig. 8D.

To circumvent the interlocking behaviour with corrugated surfaces, we implemented multiple spines on the cylindrical cage. Even if one spine aligns and interlocks with a structured surface, a second spine is likely to engage properly as they have different orientations. The flexibility and dynamics of a flying platform is also a factor that limits the probability of perfect alignment. This was corroborated by our experience as we did not experience such behaviour when landing on corrugated structures.

\section{Demonstration}

For the flight tests, HEDGEHOG was equipped with a commercially available F405 flight controller. From establishing a contact (Fig. 2C) to reaching a stable perching position (Fig. 2D), we gradually reduced the thrust while at the same time reducing the PID parameters of the controller to achieve a smooth transition, as otherwise the flight controller tries to counteract the tilting of the drone causing unwanted oscillations.

We have performed both indoor and outdoor tests. The indoor tests were made on horizontal and inclined smooth, circular wood rods of diameter $15 \mathrm{~mm}$. With manually controlling HEDGEHOG, we were capable to perch on the horizontal rods and on inclined rods up to an angle of $\pm 15^{\circ}$ and subsequently take off, as shown in Fig. 9. Beyond $\pm 15^{\circ}$, HEDGEHOG started rotating around the engaged spine when the thrust was reduced and would eventually disengage. It must 


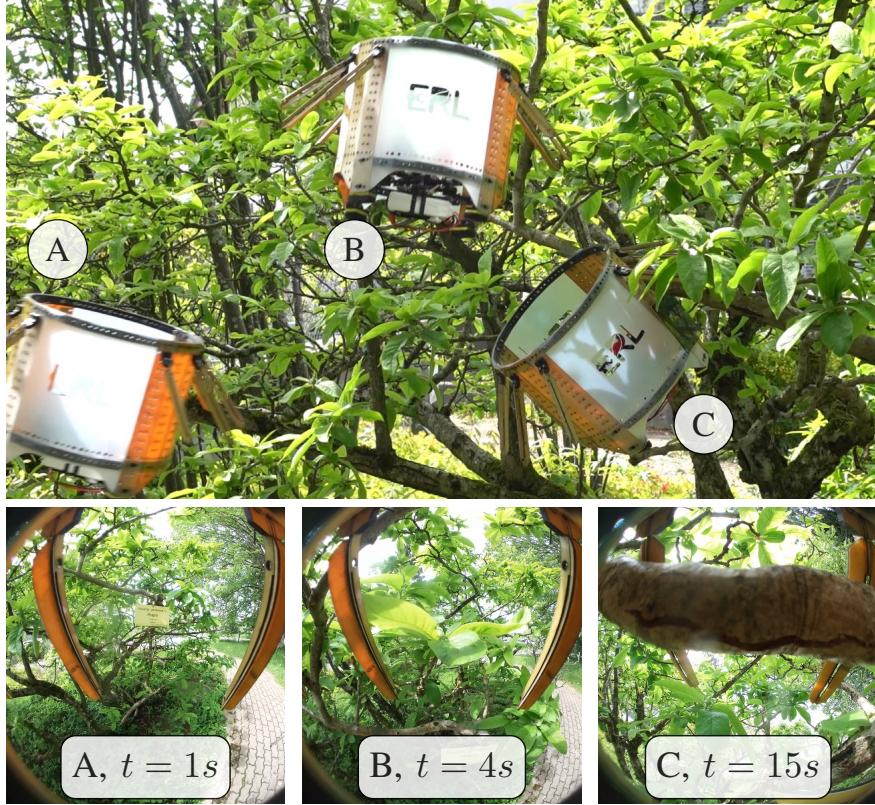

Fig. 10. Perching sequence on a tree branch from side view (top) and onboard camera (bottom). HEDGEHOG is (A) approaching, (B) positioning so that spines are above the perching object and $(\mathrm{C})$ reaching a stable position on the tree branch after spine engagement.

be noted that this angle depends on the friction coefficient. For our tests, the smooth rod had a friction coefficient close to smooth MDF from Table I. For the outdoor tests, HEDGEHOG successfully perched on different sized branches as illustrated in Fig. 1. A sequence from approach to reaching a stable perching position is shown in Fig. 10. The on-board camera shows the opening of the spines as weight is transferred onto them while lowering down the UAV. HEDGEHOG could take off after perching by increasing thrust to disengage the spines, effectively reversing the steps described in Fig. 2.

\section{CONCLUSiON}

We have developed a passive perching mechanism that can attach to overhanging branches. By employing origami technology, we have created a lightweight system that can be integrated on small aerial vehicles, as shown with HEDGEHOG. A single origami spine with a weight of only $5 \mathrm{~g}$ can sustain a $400 \mathrm{~g}$ drone and grants it the ability to perch. We demonstrated successful perching with HEDGEHOG in controlled indoor and natural outdoor environments. Our platform was able to attach to tree branches with diameters up to $86 \mathrm{~mm}$ and inclinations of $\pm 15^{\circ}$ and completely turn off the motors to conserve power.

HEDGEHOG could be improved by implementing retractable spines and optimizing the protective cage to reduce drag during flight. For automation, a control and perception strategy is required. As the mechanism does not require precise alignment with the environment, an autonomous behaviour could be based on visually detecting suitable inclined tree branches followed by a controlled approach and descent maneuver. However, the biggest challenge lies in flying in an unstructured environment with wind, moving foliage and compliant branches, where small errors could lead to unexpected behavior and failure. An autonomous or semi-autonomous control strategy could facilitate perching on more inclined and compliant branches. Swinging motions caused by wind, which could otherwise cause an increase of the tilt angle and lead to unwanted disengagement, could be actively dampened at the expense of energy.

Such passive systems may increase system safety by allowing emergency landings in more diverse environments in case of low battery or changing weather conditions. By enabling drones to perch with our solution, mission times could be increased from minutes to several hours. Therefore, longer term environmental monitoring, data collection and observation tasks in man-made and natural environments using aerial robots are within reach. The larger spatial and temporal data collection could be a basis for a holistic approach to climate change mitigation, ecosystem conservation and restoration.

\section{APPENDIX A}

From Fig. 2E, the following force relationships can be shown:

$$
\begin{aligned}
F_{N} & =F_{g} \cos (\theta), \\
F_{l} & =F_{g} \sin (\theta), \\
F_{\mu} & =\mu F_{N} .
\end{aligned}
$$

For the spine not to slip, the friction force $F_{\mu}$ needs to be larger than the load $F_{l}$ along the spine:

$$
F_{\mu} \geq F_{l} \text {. }
$$

With equations $(3,4,5)$, this yields:

$$
\mu \geq \tan (\theta) .
$$

The tilt angle $\theta$ of the platform during perching can be derived from geometric relationships shown in Fig. 2E. The angle $\theta$ can be described in the right-angled triangle "spine (orange) - vertical axis (red) - horizontal axis (red)". The angle between $F_{g}$ and $F_{l}$ is denoted as $\epsilon$. Thus

$$
\theta=90^{\circ}-\epsilon .
$$

$\epsilon$ can be described in the triangle "spine (orange) - cage (grey) - vertical axis (red)" through:

$$
\epsilon=180^{\circ}-\gamma-\delta,
$$

with $\delta$ being the angle between the protective cage, described by length $H$, and the vertical axis (red). $\delta$ can be further expressed as a function of $\beta$ and its partial angles $\beta_{1}$ and $\beta_{2}$, established between the vertical axis (red) and diagonal $D$ (dashed black) and radius $R$ (black) respectively:

$$
\delta=90^{\circ}-\beta_{2}=90^{\circ}-\left(\beta-\beta_{1}\right) .
$$

With the length $l_{e}$ and the diagonal $D$, we can calculate $\beta_{1}$ in the triangle "diagonal (dashed black) - vertical axis (red) spine (orange)" through:

$$
\tan \left(\beta_{1}\right)=\frac{l_{e}}{D},
$$

with $D=\sqrt{H^{2}+R^{2}}$. Together with equation (10), this yields the tilt angle as:

$$
\theta=\gamma-\beta+\arctan \left(l_{e} / D\right)
$$



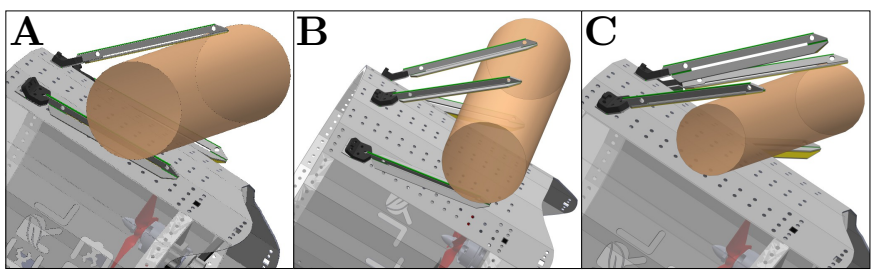

Fig. 11. Perching configurations on a cylindrical, straight branch: (A) Single spine engaged with adjacent spines folded under the branch, (B) two spines engaged with adjacent spines folded or (C) two spines engaged with a nonengaged spine in between.

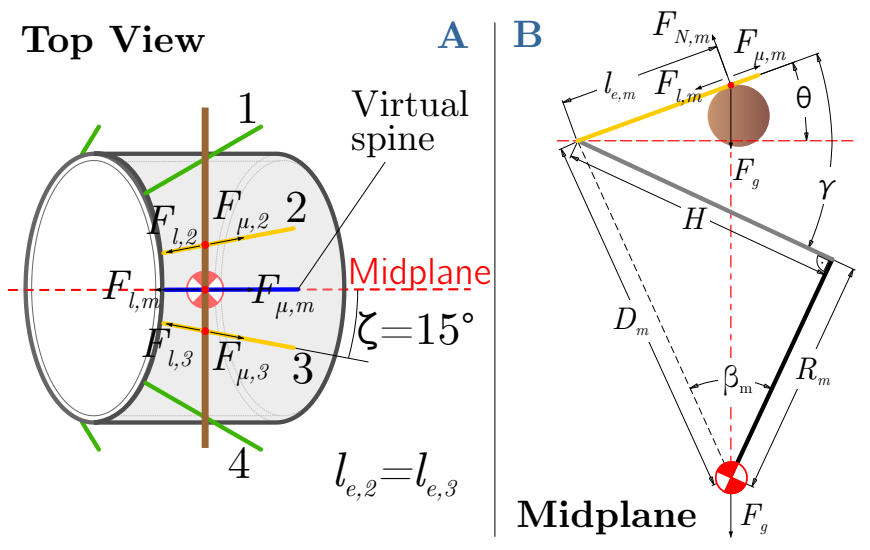

Fig. 12. Two symmetrically engaged spines on a horizontal branch. (A) Top View with spines 2 and 3 engaged, (B) Midplane in which the virtual spine and thus the relevant tilt angle and forces are defined.

\section{APPENDIX B}

Four spines are integrated circumferentially $30^{\circ}$ apart on the front and back of the cage. With multiple spines, the chance of passively engaging with a branch is increased. When engaging with two spines, the load can be distributed from two points of engagement and a more stable perch can be achieved. However, at a given time a maximum of two spines can engage due to the orientation of the circumferentially spaced spines when considering a straight, cylindrical branch. As a result, a single, two adjacent spines or two spines with a non-engaged spine in between can engage, shown in Fig. 11.

A simplified model considering two symmetrically engaged spines $n=2,3$ on a horizontal branch is shown in Fig. 12A. A virtual spine in the midplane between the two engaged spines is established. The relevant tilt angle $\theta$ of the platform is defined in this midplane. The individual forces along the spines $n=2,3$ can be projected onto the virtual spine in the midplane with $\zeta$ as defined in Fig. 12:

$$
\begin{aligned}
F_{l, m} & =F_{l, n} \cos (\zeta), \\
F_{\mu, m} & =F_{\mu, n} \cos (\zeta) .
\end{aligned}
$$

The dependency of the friction coefficient $\mu$ on angle $\zeta$ cancels out when inserting $F_{l, m}$ and $F_{\mu, m}$ into eq. (6), thus still yielding the relationship from equation (7). By taking $\zeta$ into account for $l_{e, m}, \beta_{m}$ and $D_{m}$ :

$$
\begin{aligned}
l_{e, m} & =l_{e, n} \cos (\zeta), l_{e, n}=\text { const } . \\
D_{m} & =\sqrt{H^{2}+R_{m}^{2}} \\
\beta_{m} & =\arctan \left(\frac{H}{R_{m}}\right)
\end{aligned}
$$

$$
\text { with } n=2,3 ; R_{m}=R \cos (\zeta) \text {, }
$$

eq. (2) can also be applied to the virtual spine model (Fig. 12) for two symmetrically engaged spines. In section III-B, we show that this leads to a reduced tilt angle for a given $l_{e, n}$ when two spines are engaged, thus making this engagement beneficial.

\section{REFERENCES}

[1] J.-F. Bastin, Y. Finegold, C. Garcia et al., "The global tree restoration potential," Science, vol. 365, no. 6448, pp. 76-79, 2019.

[2] M. Lowman, S. Devy, and T. Ganesh, Treetops at Risk, M. Lowman, S. Devy, and T. Ganesh, Eds. Springer New York, 2013.

[3] E. M. Mrema, T. W. Crowther, W. Mathai et al., "Ten years to restore a planet," One Earth, vol. 3, no. 6, pp. 647-652, 2020.

[4] A. Nakamura, R. L. Kitching, M. Cao et al., "Forests and Their Canopies: Achievements and Horizons in Canopy Science," Trends in Ecology \& Evolution, vol. 32, no. 6, pp. 438-451, 2017.

[5] E. Bennitt, H. L. A. Bartlam-Brooks, T. Y. Hubel et al., "Terrestrial mammalian wildlife responses to Unmanned Aerial Systems approaches," Scientific Reports, vol. 9, no. 1, p. 2142, 2019.

[6] M. Mulero-Pázmány, S. Jenni-Eiermann, N. Strebel et al., "Unmanned aircraft systems as a new source of disturbance for wildlife: A systematic review," PLOS ONE, vol. 12, no. 6, 2017.

[7] Y. Mulgaonkar, A. Makineni, L. Guerrero-Bonilla et al., "Robust Aerial Robot Swarms Without Collision Avoidance," IEEE Robotics and $\mathrm{Au}$ tomation Letters, vol. 3, no. 1, pp. 596-603, 2018.

[8] C. H. Tan, J. Tze Huan Goh, W. J. Ang et al., "Design and development of micro-aerial vehicle for tree inspections," in 2017 IEEE International Conference on Cybernetics and Intelligent Systems (CIS) and IEEE Conference on Robotics, Automation and Mechatronics (RAM). IEEE, 2017, pp. 593-598.

[9] P. Zheng, X. Tan, B. B. Kocer et al., "TiltDrone: A Fully-Actuated Tilting Quadrotor Platform," IEEE Robotics and Automation Letters, vol. 5, no. 4, pp. 6845-6852, 2020.

[10] M. Kovač, J. Germann, C. Hürzeler et al., "A perching mechanism for micro aerial vehicles," Journal of Micro-Nano Mechatronics, vol. 5, no. 3-4, pp. 77-91, 2009.

[11] A. Lussier Desbiens, A. T. Asbeck, and M. R. Cutkosky, "Landing, perching and taking off from vertical surfaces," The International Journal of Robotics Research, vol. 30, no. 3, pp. 355-370, 2011.

[12] M. T. Pope, C. W. Kimes, H. Jiang et al., "A Multimodal Robot for Perching and Climbing on Vertical Outdoor Surfaces," IEEE Transactions on Robotics, vol. 33, no. 1, pp. 38-48, 2017.

[13] L. Daler, A. Klaptocz, A. Briod et al., "A perching mechanism for flying robots using a fibre-based adhesive," in 2013 IEEE International Conference on Robotics and Automation. IEEE, 2013, pp. 4433-4438.

[14] A. Kalantari, K. Mahajan, D. Ruffatto et al., "Autonomous perching and take-off on vertical walls for a quadrotor micro air vehicle," in 2015 IEEE International Conference on Robotics and Automation (ICRA). IEEE, 2015, pp. 4669-4674.

[15] E. W. Hawkes, H. Jiang, and M. R. Cutkosky, "Three-dimensional dynamic surface grasping with dry adhesion," The International Journal of Robotics Research, vol. 35, no. 8, pp. 943-958, 2016.

[16] K. Zhang, P. Chermprayong, T. M. Alhinai et al., "SpiderMAV: Perching and stabilizing micro aerial vehicles with bio-inspired tensile anchoring systems," in 2017 IEEE/RSJ International Conference on Intelligent Robots and Systems (IROS). IEEE, 2017, pp. 6849-6854.

[17] M. Pope, "Microspines Make It Easy for Drones to Perch on Walls and Ceilings," IEEE Spectrum, 2016. [Online]. Available: http://spectrum.ieee.org/automaton/robotics/drones/ microspines-make-it-easy-for-drones-to-perch-on-walls-and-ceilings

[18] K. Yanagimura, K. Ohno, Y. Okada et al., "Hovering of MAV by using magnetic adhesion and winch mechanisms," in 2014 IEEE International Conference on Robotics and Automation (ICRA). IEEE, 2014, pp. $6250-6257$

[19] K. Hang, X. Lyu, H. Song et al., "Perching and resting-A paradigm for UAV maneuvering with modularized landing gears," Science Robotics, vol. 4 , no. $28,2019$.

[20] W. Chi, K. H. Low, K. H. Hoon et al., "An optimized perching mechanism for autonomous perching with a quadrotor," in 2014 IEEE International Conference on Robotics and Automation (ICRA). IEEE, 2014, pp. 3109-3115. 
[21] W. R. T. Roderick, H. Jiang, S. Wang et al., "Bioinspired Grippers for Natural Curved Surface Perching," in Lecture Notes in Computer Science. Springer Verlag, 2017, pp. 604-610.

[22] M. A. Graule, P. Chirarattananon, S. B. Fuller et al., "Perching and takeoff of a robotic insect on overhangs using switchable electrostatic adhesion," Science, vol. 352, no. 6288, pp. 978-982, 2016.

[23] H.-N. Nguyen, R. Siddall, B. Stephens et al., "A Passively Adaptive Microspine Grapple for Robust, Controllable Perching," in 2019 2nd IEEE International Conference on Soft Robotics (RoboSoft). IEEE, 2019 , pp. $80-87$.

[24] S. W. Chen, G. V. Nardari, E. S. Lee et al., "SLOAM: Semantic Lidar Odometry and Mapping for Forest Inventory," IEEE Robotics and Automation Letters, vol. 5, no. 2, pp. 612-619, 2020. 